\title{
A MYSTERIOUS UNIVERSE \\ REVEALING THE BRIGHT AND DARK SIDES OF THE COSMOS
}

SuSANA PlanELles AND VERONICA BIFFI

Why is our universe as we observe it? Will it be the same forever? Understanding the nature of the main constituents of the universe is crucial to obtain a precise description of the way in which it reached its present state. Nowadays, many independent observations support a picture in which the matter content of the universe is shared between an ordinary and observable baryonic component $(\sim 5 \%)$ and an invisible dark matter ( $23 \%)$. The remaining $\sim 72 \%$ of the universe content is in the form of a completely mysterious dark energy field. This composition emphasizes that, while $\sim 95 \%$ of our universe represents a major uncertainty for us, even the minor contribution from normal and, apparently, known matter entails important challenges for cosmologists.

Keywords: cosmology, astrophysics, baryons, dark matter, dark energy.

\section{ONLY MATTER OR SOMETHING MORE?}

Our comprehension of the universe has changed dramatically in the last hundred years. At the beginning of the past century, our galaxy, the Milky Way, was thought to be the centre of our universe. Beyond the extension of our galaxy, everything was uncertain. By then, a number of nebulae had been already observed but they were believed to be unresolved groups of stars belonging to our galaxy. In 1924, Edwin Hubble measured the distance to some of these nebulae demonstrating that, in fact, they were galaxies placed far away from the limits of the Milky Way, confirming therefore the existence of the extragalactic universe.

Some years before, in 1915, Albert Einstein presented his theory of general relativity, a unified and revolutionary description of gravity as a property of space-time. When in 1925 Alexander Friedmann resolved Einstein's equations under the conditions of homogeneity and isotropy on large scales, he obtained the description of an expanding universe!
Only four years later, analysing the motions of nearby galaxies, Hubble discovered a nice correlation: the further the galaxies were from each other, the higher were their recession velocities. Since galaxies were not moving around a common centre, this result suggested that, in the past, all galaxies would have been closer to each other and, therefore, had a common origin. These observations provided the first confirmation of an expanding universe with a common starting point, the Big Bang.

The current standard cosmological model, based on the Big Bang theory, was definitely established as a valid description of our universe in 1965, when Arno Penzias and Robert Wilson discovered the first light emitted from the universe after the Big Bang, the cosmic microwave background radiation, detected as an isotropic and unpolarised emission filling all the space.

The Big Bang theory succeeds in explaining many observational evidences (Hamilton, 2013). According to this model, our universe and, therefore, the spacetime began after the Big Bang around 13,770 million years ago. At the beginning, all the matter, energy and 
radiation were compressed into a very hot and dense plasma. Given the high temperatures, the matter was completely ionized and the photons interacted strongly with the electrons. As the universe expanded, it cooled down until reaching a temperature in which the electrons were captured by atomic nuclei to form the first atoms, mainly hydrogen atoms.

The afterglow of the initial hot and dense condition of the primordial universe, where matter and radiation were coupled, can be detected in the form of the microwave radiation that fills uniformly the sky, namely the cosmic microwave background. This radiation, emitted when the universe was around 375,000 years old, pictures a very homogeneous medium with an extremely uniform temperature across the sky, in which only tiny fluctuations can be measured. Indeed, in the last thirty years, thanks to space satellites such as COBE, WMAP or, more recently, Planck, we have been able to measure cosmic microwave background

"THE CURRENT STANDARD
COSMOLOGICAL MODEL,
BASED ON THE BIG BANG
THEORY, WAS DEFINITELY
ESTABLISHED AS A
VALID DESCRIPTION OF
OUR UNIVERSE IN 1965,
WHEN THE COSMIC
MICROWAVE RADIATION WAS
DISCOVERED»

temperature anisotropies with an impressive precision (Figure 1). These anisotropies, a consequence of the tiny density fluctuations filling the primordial universe, represent the seeds for the formation of the cosmic structures: due to the effect of gravity, those initial over-dense regions in the space gave rise to the largest systems we observe today.

Through the investigation of the cosmic microwave background temperature fluctuations,

cosmologists have become very close to answer one of the most fundamental questions: how did all the things in our present-day universe come to be the way they are? Indeed, these and many other observations have revealed an interesting and surprising fact: most of the matter in the universe is not visible in the classic sense but dark, since it does not emit electro-magnetic waves. The dark matter can still be «observed» indirectly via its effects on and interaction with the visible structures. Other than matter, we know that the universe is also

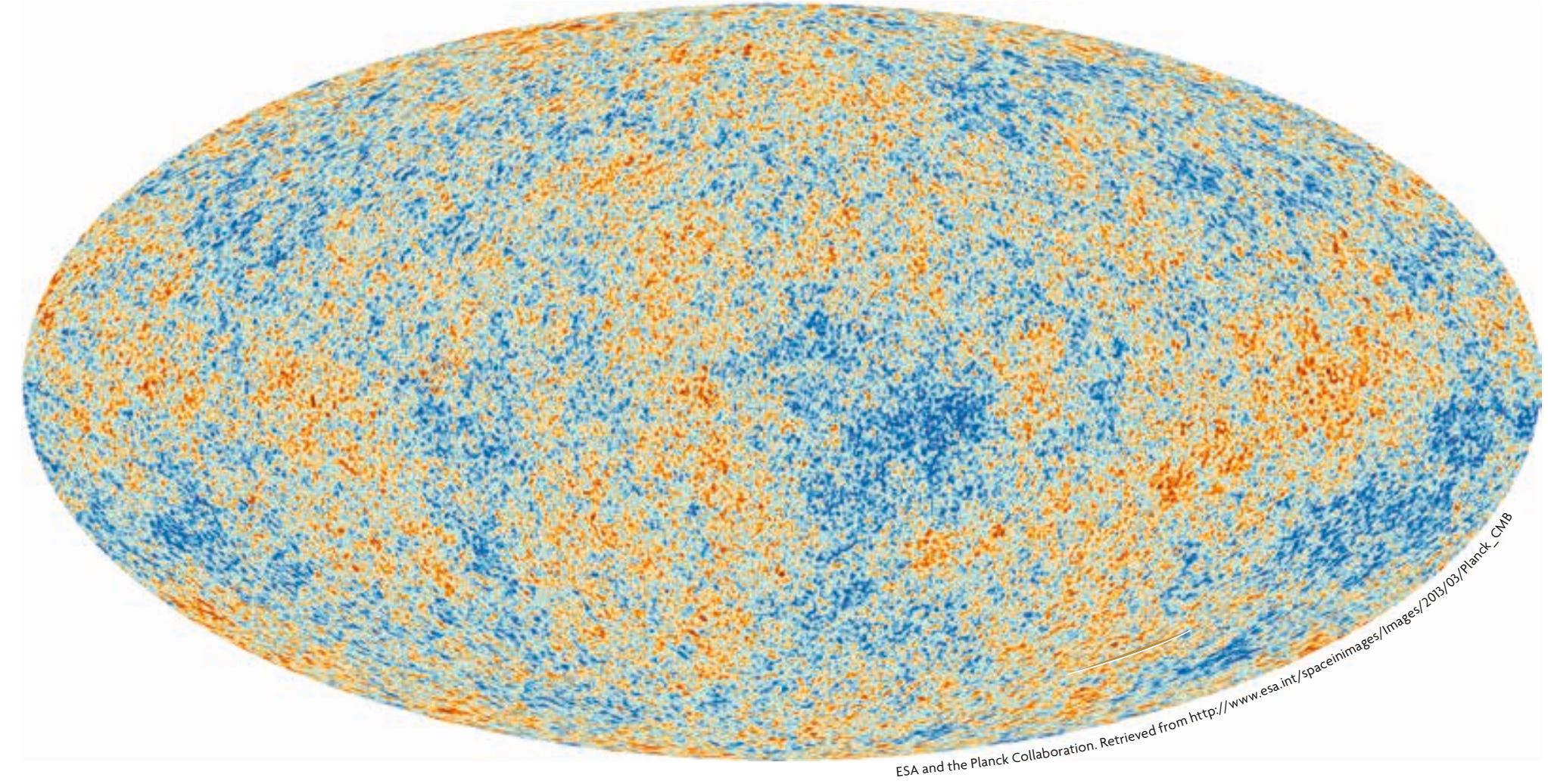

Figure 1. Distribution of cosmic microwave background temperature fluctuations as observed by the Planck satellite. This map represents a picture of the light emitted by the early universe, when it was 380,000 years old. Redder colours correspond to hotter and, therefore, denser regions in space that represent the seeds for the formation of the cosmic structures we observe today. 


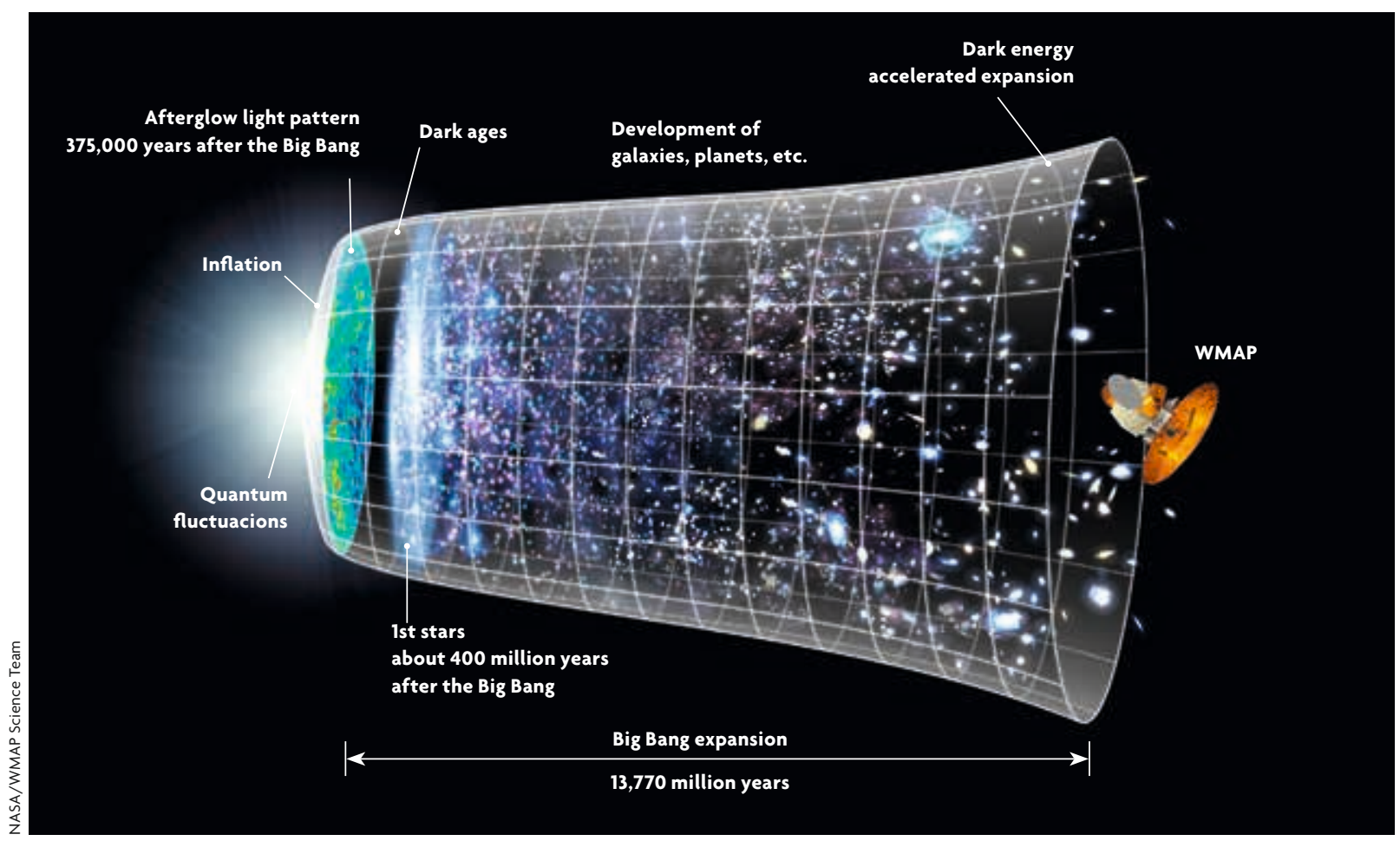

Figure 2. Illustrative diagram summarising the currently accepted standard cosmological model. The image shows the evolution of the universe from the Big Bang, around 13,770 million years ago, to the present day.

permeated with radiation. All this, however, is just a minor fraction of the present-day universe content. Although the energy density of the various components in the universe changes with time, during the present epoch, while we observe it, the dominant component of the energy density is in the form of a mysterious and unknown dark energy field.

Very recently, on September 2015, gravitational waves, that is, ripples in space-time generated in this case by the merger of two black holes, have been detected by the LIGO observatories, confirming in an excellent way the prediction made, almost a century ago, by the theory of general relativity and supporting our current cosmological model (Figure 2).

\section{BARYONIC MATTER: CHARTING THE VISIBLE}

Astronomical observations have revealed a universe in which cosmic structures grow and evolve in a hierarchical way, giving rise to a number of objects over a wide range of masses and scales. During cosmic evolution, gravitational attraction makes stars to collapse into galaxies, and galaxies into larger systems known as galaxy clusters. This large scale structure of the universe is completed by a network of less-dense filaments connecting galaxy clusters, and almost empty regions or «voids». Large galaxy surveys, such as the Sloan Digital Sky Survey, have confirmed this picture with an unprecedented precision (e.g., Bull et al., 2016; Olmo, 2012).

Measurements from cosmic microwave background anisotropies and from early nucleosynthesis have revealed that in the early universe ordinary matter accounts for $5 \%$ of its total mass-energy content. Stars, planets, clouds of gas and dust are formed by this baryonic matter, which is composed by ordinary atoms and ions and is observed to emit radiation over a wide range of energies. However, one open question related with this ordinary component is that, when astronomers sum up all the visible matter at the present epoch, a fraction of more than half of the baryons is missing. More specifically, the present baryonic content of the universe is contributed by galaxies $(\sim 10 \%)$, warm gas in the space $(\sim 10 \%)$ and clouds of cold gas in the intergalactic medium (30\%). The remaining $\sim 50 \%$ remains undetected (Figure 3).

Cosmological simulations suggest that a large fraction of these missing baryons resides in a warm-hot intergalactic medium distributed in the filamentary cosmic web. This diffuse and hot plasma, formed by highly ionized material, is challenging to be detected by current observational facilities. However, recent X-ray observations of 


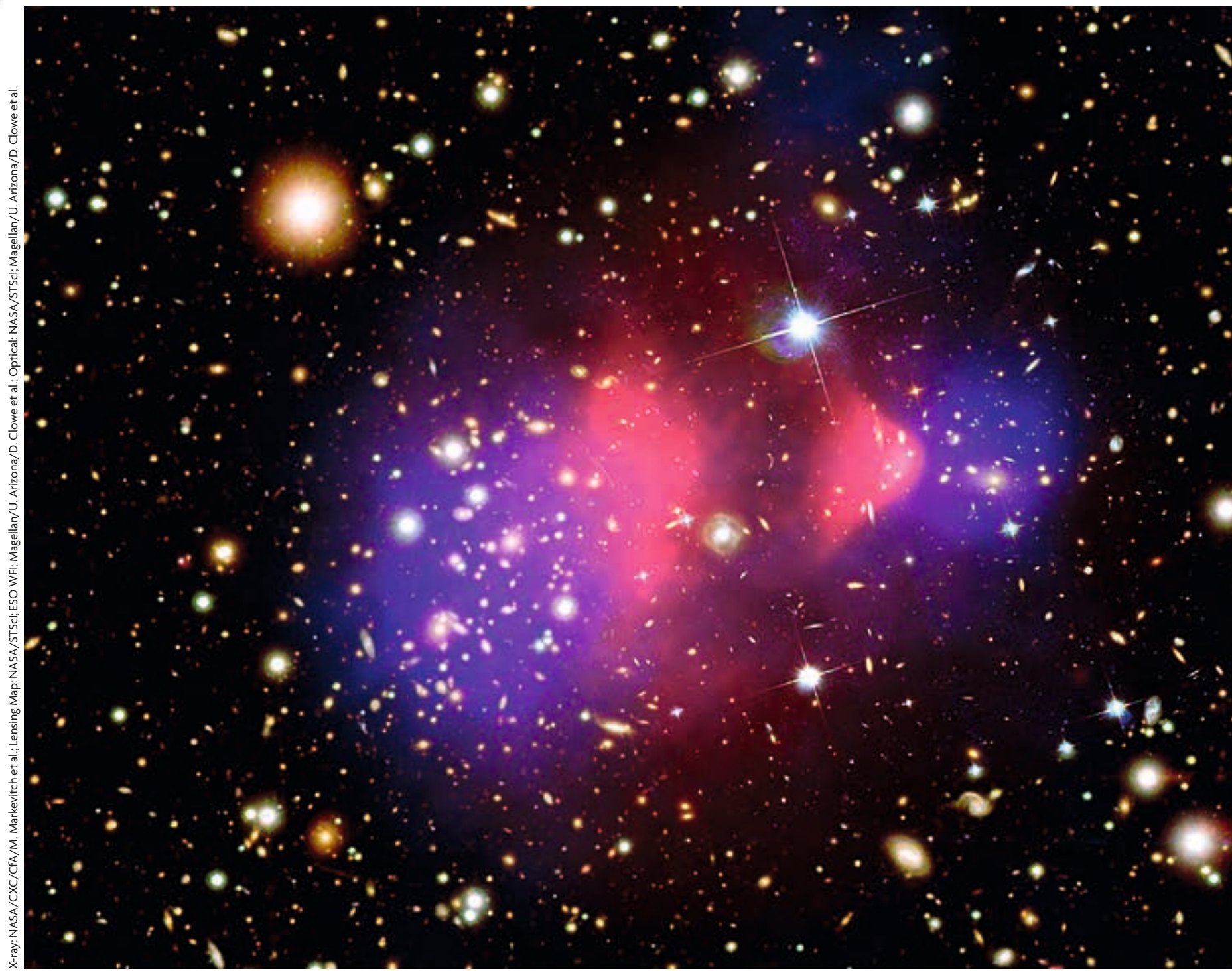

filaments associated to the massive galaxy cluster Abell 2744 seem to support the picture suggested by simulations (Eckert et al., 2015). At the same time, an analysis of the distortions experimented by the cosmic microwave background radiation when it passes through the central galaxies identified in the Sloan Digital Sky Survey has claimed to detect all the missing baryons within and around the galaxies (Hernández-Monteagudo et al., 2015). These results indicate that the mystery of missing baryons, although quite close to be resolved, is still controversial.

Although the fraction of missing baryons is consistent with reasonable models of the impact of galaxy winds in the standard cosmological model, detecting this matter component remains an important challenge in order to understand the composition of our universe and how it came to be what we observe today.
Figure 3. Distribution of matter in the galaxy cluster IE 0657-56, commonly known as the Bullet Cluster. This structure results from the collision of two subclusters, being the smaller one, on the right side of the image, referred to as the «bullet" that has just passed the larger subcluster. The baryonic content of this cluster is distributed between individual galaxies, observed in the optical band, and the hot intra-cluster gas emitting in X-rays and represented here by the pink distributions. Thanks to the gravitational lensing effect of background galaxies, we know that most of the matter in this cluster is in the form of dark matter, represented here by the blue clouds. The separation between the gas and dark matter distributions, which only interact gravitationally, provides strong evidence for the existence of dark matter. 


\section{DARK MATTER: SEARCHING FOR THE INVISIBLE}

The cosmic microwave background observations predict that around $80 \%$ of the matter in the universe is invisible to our telescopes and detectors. We cannot observe this «dark matter» component, but we infer its existence from its gravitational interaction with normal matter (e.g., Bull et al., 2016).

In 1933, from the analysis of the dynamics of galaxies in the Coma Cluster, the astronomer Fritz Zwicky realized that the total mass in luminous galaxies was much smaller than the total gravitational mass of the cluster. To explain this discrepancy, he suggested the presence of a dominant, undetected and invisible dark matter filling the space between galaxies.

However, the existence of dark matter was not commonly accepted until more than forty years later, when Vera Rubin and her collaborators obtained a similar result from observations of the rotational curves of spiral galaxies. Contrarily to what happens in a pure Newtonian universe, they observed a constant stellar velocity in the outer regions of galaxies, confirming the existence of a dark matter filling the galactic potential and keeping the stars bound to the galaxy even at large galactic-centre distances.

Dark matter is also detected through the gravitational lensing effect. According to general relativity, the trajectory of light is bended when it passes close to a massive object. As a result, when the light from background galaxies travels through more massive foreground systems, such as galaxy clusters, we observe distorted and elongated galaxy images. The magnitude of this effect depends on the total mass of the cluster acting as a lens and provides one of the most precise estimations of cluster masses (Figure 3).

Since 1980, a number of additional independent observations, such as the distribution of cosmic microwave background anisotropies or X-ray observations of the hot intra-cluster gas in galaxy clusters, confirmed the presence of a significant amount of dark matter in the universe.

The detection of dark matter from a variety of gravitational effects provides strong constraints on its main properties. So far, dark matter has not been observed to interact with baryons or light - other than gravitationally - and, as a consequence, it should be dark in the sense that it does not emit or absorb light. Moreover, dark matter must be cold (or, equivalently, with a very low velocity), in order to be able to collapse and allow the formation of gravitationally bound objects. Observational evidence also suggests that dark matter should be in the form of non-collisional matter. Although there are several solutions satisfying these requirements, there is a general consensus in that, if dark matter exists, it should be in the form of a new particle still missing in the standard model of particle physics.

Currently, there are a number of experiments trying to detect dark matter from different channels. For instance, experiments at the Large Hadron Collider (LHC), the largest particle accelerator, try to produce dark matter candidates by colliding known particles at different energies. Since dark matter should be stable and only weakly interacting with normal matter, detectors will only be able to measure the energy loss after collision with the particles arriving to them. Moreover, contrarily to normal matter, since dark matter particles should be continuously arriving to our planet and passing through its surface with almost no interaction, a number of underground experiments have been also built to this end. However, for the moment, both these channels have been unsuccessful.

In addition, indirect detection experiments search for dark matter annihilation processes which will produce very energetic photons such as gamma rays. Regions with high concentrations of dark matter, such as the centre of our galaxy or dwarf galaxies, are the perfect places to detect these events. Despite the number of gammaray occurrences collected since 2008 by the Fermi Gamma-ray Space Telescope, there is not a clear confirmation of these processes.

Numerical simulations accounting for the gravitational evolution of billions of dark matter particles confirm that the cold dark matter model is consistent, at least on large scales, with the distribution of cosmic structures as provided by large galaxy surveys (Figure 4). On smaller scales, however, a number of tensions between the accepted model and observations call for a more accurate modelling of different physical processes, which are mainly relevant on galactic scales (e.g., Bull et al., 2016). In this sense, the improvement of current numerical simulations - in terms of resolution and physicswill be crucial to deepen our understanding of the universe and its main constituents. 


\section{MONOGRAPH}

Violent universe

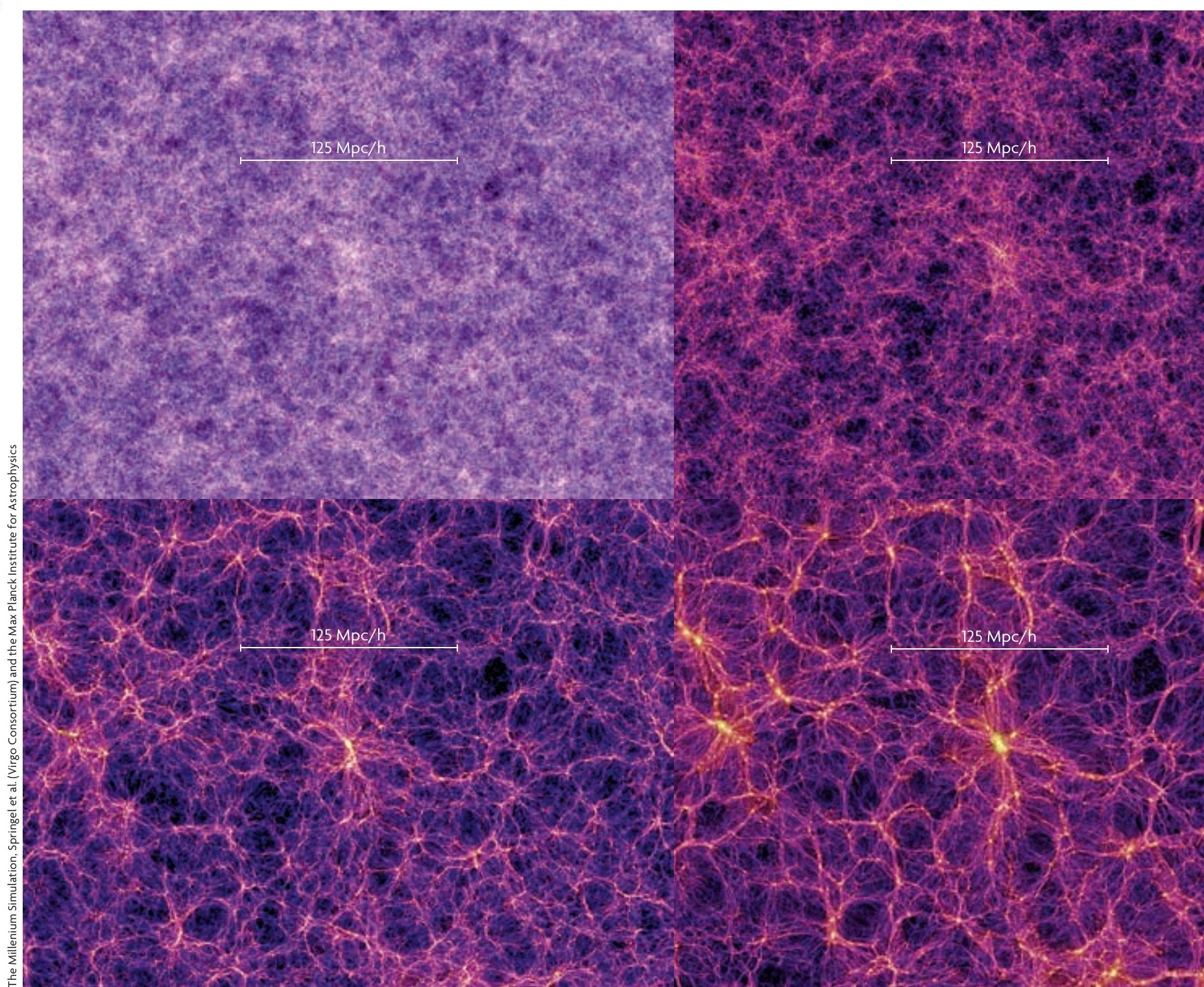

\section{DARK ENERGY. GUESSING THE UNKNOWN}

Amazingly, the vast majority of the universe energetic content is not represented by radiation or matter, neither luminous nor dark, but rather consists of a still poorly known form of energy, the so-called «dark energy». Its main characteristic is that, differently from every other known form of matter or energy, it has a negative pressure, acting therefore as a repulsive force (e.g., Bull et al., 2016).

The confirmation arrived in the late 90s when two independent studies found observational evidences of an accelerated expansion of the universe. The importance of such crucial discovery granted Saul Perlmutter, Brian P. Schmidt, and Adam G. Riess a Nobel Prize in 2011.
Figure 4. Distribution of the simulated dark matter density field in a small region of the Millenium Simulation. This simulation resolved the evolution of more than 10,000 million dark matter particles in a cubic region of the universe of around $10^{21}$ kilometres per side. Clockwise, from the upper left panel, the 2D maps show the distribution of dark matter when the universe is, respectively, $210,1,000,4,700$ and 13,600 million years old. These images exemplify the hierarchical fashion in which cosmic structures evolve. As the evolution proceeds, the matter distribution goes from an almost homogenous network of overdense regions, filaments and voids, to a less homogenous pattern where large overdense regions (in yellow), associated to present-day galaxies and galaxy clusters, are clearly distinguished. The straight line in all panels provides the scale of the images, where $125 \mathrm{Mpc} / \mathrm{h}$ is equivalent to more than $10^{19}$ kilometres. 
Both groups investigated type Ia supernovae, particularly energetic stellar explosions that mark the death of a small compact star, belonging to a binary system, with an enormous release of energy. All supernovae shine with a similar intrinsic brightness, so that depending on the observed dimming of their light, they can be used to measure distances in the universe and therefore its expansion with time. Both groups independently discovered that our universe is not slowing down, due to the gravitational attraction of the matter it contains, but it is rather expanding faster and faster. Since this result cannot be explained only with the known kinds of matter and energy, a dominating dark energy is suggested to be responsible for it. Combining this with the cosmic microwave background observation of a flat universe, the amount of dark energy required to explain current observations should be $\sim 72 \%$ of the total energy budget.

In one of the simplest interpretations, dark energy can be related to a vacuum energy, that is, the energy associated to empty space, well represented by the so-called cosmological constant. Its first appearance dates back to Einstein's formulation of the equations describing our universe. Despite the fact that a physical meaning was actually missing, he introduced this concept as a way to compensate the pull of gravity and build a «static» universe, neither expanding nor contracting with time, which was the accepted scenario by then. This picture, however, was abandoned after Hubble provided observational proofs for an expanding universe and no need for the cosmological constant.

However, since the late 90s, the various evidences for the accelerated expansion of the universe made dark energy become part of the standard cosmological model (Figure 2). For the sake of simplicity, the accepted scenario includes dark energy in the form of a cosmological constant, but a deeper understanding of its nature is still an open challenge. In fact, despite its effectiveness, it suffers from both theoretical and observational drawbacks. On the one hand, the value of such vacuum energy is measured to be positive but also so incredibly tiny, that it is hard to explain in a natural way from the point of view of particle physics. On the other hand, observational evidences for the existence of dark energy only consist of indirect proofs of its negative pressure accelerating the expansion of the universe. Furthermore, with respect to the critical density of our universe, dark energy started to dominate, over matter and radiation, the energetic budget of the universe and to drive its accelerated expansion only very recently in the cosmic time. Why only now? This also raises an embarrassing open question to cosmologists.

\section{WHAT IS BEHIND DARK ENERGY?}

A whole branch of cosmology is devoted to investigate the nature of dark energy, both observationally and theoretically and, besides the cosmological constant, many models have been proposed to explain the increasing amount of precision cosmological data.

How to choose among all of them? If more than one model represents successfully part of the observed properties of the universe, which are the ways to discriminate? Scientists typically invoke the criteria of simplicity and naturalness. This is the main reason why the cosmological constant is still the favoured one.

Among the various alternatives, there are complicated allencompassing theories that attempt to interpret both dark matter and dark energy as two aspects of a single phenomenon that modifies gravity at various spatial scales. Either $72 \%$ of the universe consists of the mysterious dark energy, or general relativity must be replaced by a new form of gravity on cosmic scales. This latter interpretation has been proposed by some theorists, who consider both dark energy and cosmic acceleration as a failure of general relativity on scales larger than super-clusters (i.e., large associations of groups and galaxy clusters). However, most of the attempts to modify general relativity in this perspective turned out to be inconsistent with many observational data and have been therefore ruled out.

Recently, a new campaign of type Ia supernovae observations added very precise measurements of the universe expansion rate, showing some tension with the value obtained from cosmic microwave background radiation data (Moskowitz, 2016; Riess et al., 2016). The plot thickens: apparently, the universe is expanding 


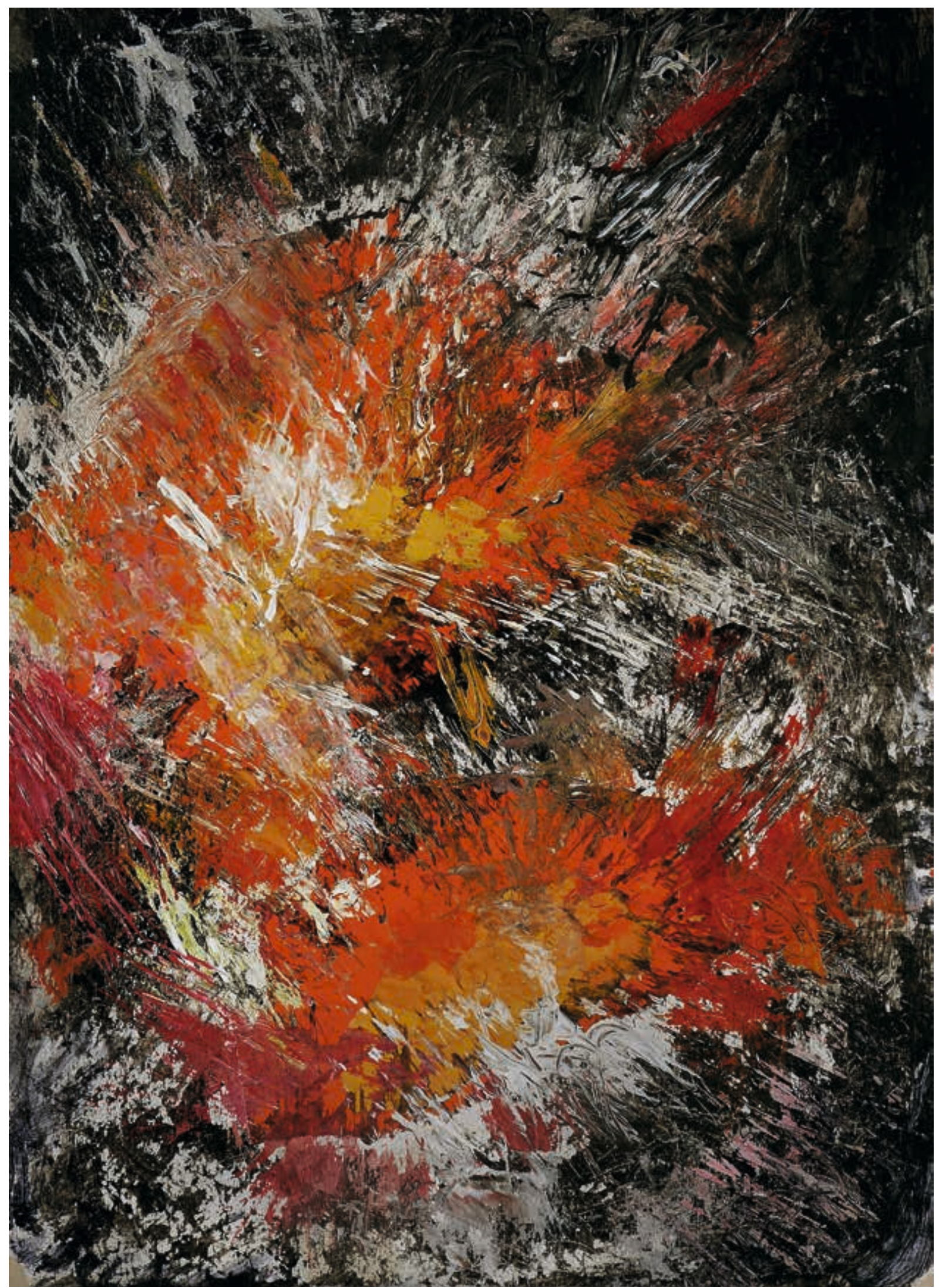

Aurora Valero. Explosions, 2016. Mixed technique, collage on paper, $25 \times 35 \mathrm{~cm}$. 
even faster than we thought. Dark energy -if it exists at all-might behave differently than speculated. For instance, this could support the idea of a dynamic dark energy changing in space and time, as the one proposed by the so-called «quintessence» models.

In this context, sophisticated cosmological simulations of the universe performed on highly advanced supercomputers, through massive and complex numerical calculations, play a crucial role. These allow to change the gravitational description of the universe and its expansion history in order to derive measurable properties of the resulting numerical structures, such as the number of systems expected at any given mass scale, that can be ultimately compared against the observed ones. This is a very useful way to test the predictions of these theories and even discard some of them.

Observationally, the chasing of something invisible and unknown, like dark energy, is always an extremely challenging and exciting mission. To tackle this ambitious task, large survey programmes designed to observe a huge amount of galaxies and supernovae out to very large distances have been planned in the recent years. Among these, the Dark Energy Survey and the Euclid mission have the primary scope of probing the geometry and expansion of the universe by looking at the details of the galaxy distribution on large scales and measuring the tiny effects of gravitational lensing induced by the dark matter distribution. These measurements will cover the cosmic period over which dark energy started to drive the cosmic acceleration and therefore will provide more insights on the intrinsic nature of dark energy and help to answer many open questions: is the dark energy a constant over time? Why did it start to dominate only recently? What is it? Does it really exist or is it our understanding of gravity that must be changed?

\section{LOOKING TO THE FUTURE}

In less than one century, thanks to the combination of observational, numerical and theoretical developments, an impressive progress has been made in our understanding of the universe. Despite the remarkable achievements, cosmology still depicts an optimal and fertile ground for new theories and alternative models to develop, sometimes turning into philosophical questions but continuously pushing the scientific progress forward. In fact, every successful theory and every observational discovery always bring new challenges and new open windows on the universe. Adding pieces to the whole picture is crucial not only to understand the universe we observe today, but also and, especially, to deepen our understanding of its origin and ultimate fate.

By the time we are writing this article, a second gravitational wave signal has been detected, confirming a new powerful channel to explore our universe. This fact, in combination with impressive future observational facilities, such as the Square Kilometre Array, will provide us with clues to address some of the remaining open questions. What we can learn from this new window to the universe still remains a mystery. Which will be the new questions arising from these developments represents the most exciting part. (†)

\section{REFERENCES}

Bull, P., Akrami, Y., Adamek, J., Baker, T., Bellini, E., Beltrán Jiménez, J., ... Winther, H. A. (2016). Beyond $\Lambda$ CDM: Problems, solutions, and the road ahead. Physics of the Dark Universe, 12, 56-99. doi: 10.1016/j. dark.2016.02.001

Eckert, D., Jauzac, M., Shan, H., Kneib, J. P., Erben, T., Israel, H., ... Tchernin, C. (2015). Warm-hot baryons comprise 5-10 per cent of filaments in the cosmic web. Nature, 528, 105-107. doi: 10.1038/nature16058

Hamilton, J. Ch. (2013). What have we learned from observational cosmology? Studies in History and Philosophy of Science Part B: Studies in History and Philosophy of Modern Physics, 46(A), 70-85. doi: 10.1016/j. shpsb.2013.02.002

Hernández-Monteagudo, C., Ma, Y.-Z., Kitaura, F. S., Wang, W., GénovaSantos, R., Macías-Pérez, J., \& Herranz, D. (2015). Evidence of the missing baryons from the kinematic Sunyaev-Zeldovich effect in Planck data. Physical Review Letters, 115(19). doi: 10.1103/PhysRevLett.115.191301 Moskowitz, C. (2016, April 11). Cosmic speed measurement suggests dark energy mystery. Scientific American. Retrieved from http://www. scientificamerican.com/article/cosmic-speed-measurement-suggestsdark-energy-mystery/

Olmo, G. J. (2012). Open questions in cosmology. Rijeka: InTech. doi: $10.5772 / 45746$

Riess, A. G., Macri, L. M., Hoffmann, S. L., Scolnic, D., Casertano, S., Filippenko, A. V., ... Foley, R. J. (2016). A $2.4 \%$ determination of the local value of the Hubble constant. The Astrophysical Journal, 826(1). doi: $10.3847 / 0004-637 \mathrm{X} / 826 / 1 / 56$

Susana Planelles. Postdoctoral researcher at the University of Valencia (Spain), where she also obtained her PhD in 2011. Her scientific interests are mainly focused on the field of Computational Cosmology. In particular, a major part of her research is devoted to analyse and interpret the formation and evolution of galaxy clusters in large cosmological simulations.

Veronica Biffi. Postdoctoral researcher at the University of Trieste and associated to the INAF Observatory of Trieste (Italy). Starting with her $\mathrm{PhD}$ in Munich, her research mainly focuses on the physics of galaxy clusters, through their study via numerical cosmological simulations and the comparison to X-ray synthetic observations of the hot intra-cluster medium. 